\title{
Freely-Available, True-Color Volume Rendering Software and Cryohistology Data Sets for Virtual Exploration of the Temporal Bone Anatomy
}

\author{
Lüder Alexander Kahrs Robert Frederick Labadie \\ Department of Otolaryngology, Head and Neck Surgery, Vanderbilt University Medical Center, \\ Nashville, Tenn., USA
}

\section{Key Words}

Histology · Temporal bone anatomy · Three-dimensional viewer

\begin{abstract}
Background: Cadaveric dissection of temporal bone anatomy is not always possible or feasible in certain educational environments. Volume rendering using CT and/or MRI helps understanding spatial relationships, but they suffer in nonrealistic depictions especially regarding color of anatomical structures. Freely available, nonstained histological data sets and software which are able to render such data sets in realistic color could overcome this limitation and be a very effective teaching tool. Methods: With recent availability of specialized public-domain software, volume rendering of true-color, histological data sets is now possible. We present both feasibility as well as step-by-step instructions to allow processing of publicly available data sets (Visible Female Human and Visible Ear) into easily navigable 3-dimensional models using free software. Results: Example renderings are shown to demonstrate the utility of these free methods in virtual exploration of the complex anatomy of the temporal bone. After exploring the data sets, the Visible Ear appears more natural than the Visible Human. Conclusion: We provide directions for an easy-to-use, open-source software in conjunction with freely available histological data sets. This work facilitates self-education of spatial relationships of anatomical structures inside the human temporal bone as well as it allows exploration of surgical approaches prior to cadaveric testing and/or clinical implementation.
\end{abstract}

Copyright $(2013$ S. Karger AG, Basel

\section{Background}

Teaching anatomy and learning safe surgical approaches to the temporal bone are challenging because of the high density of critical anatomical structures embedded within. Especially difficult to appreciate are the narrow access paths and small dimensions required to 
reach the middle and inner ear for surgical interventions. This necessitates years of training, strong experience, and an innate sense of spatial relationships. During medical education, hand drawings, videos, enhanced photographs from microscopes or endoscopes, and imaging (e.g. CT or MRI) are used to help discern 3-dimensional (3D) relationships from 2-dimensional images $[1,2]$. Complementing this is specialized training for surgical residents during temporal bone laboratory courses using cadaveric bones as well as hands-on learning under the close supervision of an expert as is only achievable in the operating room $[3,4]$. These courses are considered the penultimate training second only to apprenticing within the operating room. Unfortunately, especially in the UK, such courses are in decline secondary to decreased availability of donated bodies [3].

Beyond standard biomedical image computing software [5], several specific computer systems have been developed to enhance teaching and understand temporal bone anatomy $[4,6,7]$. These systems provide virtual simulations $[8,9]$ some of which are enhanced with haptic feedback $[8,10-13]$. Different visualization methods and applications allow rendering of full-color image stacks such that they appear in life-like color [14-20], but there are very few which are available in the public domain and are open source [21,22]. Despite this, input data for true-color rendering histology slices (rendering of full-color images) are freely available from whole body human cryohistology [23-25]. More detailed data sets of the subregion of the ear/temporal bone are also available [26-28]. The aim of this work is to give instructions for a free method for visual exploration of otological anatomy with 3D, true-color volume rendering using these data sets.

\section{Methods}

After a critical analysis of visualization software available in the public domain, we selected the advanced volume rendering technique, introduced by Peng et al. [29, 30] formerly referred to as V3D (Viatronix Inc., Stony Brook, N.Y., USA) now freely available, including source code for nonprofit research, under the name Vaa3D. The main advantage of Vaa3D over other software is the true-color rendering capability combined with real-time interactive visualization (i.e. no render button is required and views are immediately updated after changing the view). The software runs on typical PCs and laptops with the requisite memory and a dedicated graphics card. Our test environment was a standard personal computer (laptop Lenovo Y470) with the following specifications: Intel i7 processor (2630QM, 2 GHz), 8 GB RAM and 1 GB dedicated graphics card memory (Nvidia GeForce GT550M) available for under USD 1,000. Software versions are available for download on the Vaa3D website (www.vaa3d.org). We downloaded Vaa3D version 2.707 for Windows 7 ( 64 bit). For comfortable visualization we recommend a display which can provide an application window height of at least 768 pixels.

Immediately after downloading the Vaa3D software is ready to use. Image series - i.e. multiple TIFF (tagged image file format) images - can be imported to an image stack, or image stacks (e.g. multi-page TIFF files) can be loaded directly. We tested two different data sets which include or consist of the temporal bone region: (1) the Visible Female Human [31] and (2) the Visible Ear [26]. Both data sets are nonstained histological slices and were originally acquired by cryosectioning and color photography.

(1) The Visible Human data sets are public domain (written permission is necessary prior to use) and can be downloaded via FTP from the US National Library of Medicine after receiving the necessary access data. We downloaded the '4K-Tiff-Images' of the Visible Female Human and used image file numbers 12711500 which cover the temporal bone. Using freeware (Irfanview 4.32, www.irfanview.com, free for noncommercial use) we cropped these for a left and a right ear region (fig. 1a). Both resulting image stacks have 512 $\times 512 \times 230$ voxels, and the in-plane resolution is higher $(\times 2.4)$ than in the z-direction (slice distance 0.33 $\mathrm{mm}$ ). Such anisotropy (and noninteger/non-whole-number) scaling factors need to be entered in Vaa3D. The resulting volumes cover regions of $7.04 \times 7.04 \times 7.59 \mathrm{~cm}^{3}$.

(2) The Visible Ear data set, created by Sørensen et al. [26], is published as freeware, and a CD can be ordered from Karger AG, Basel, Switzerland. The files on the CD can be copied for noncommercial research and educational purposes. The data set is comprised of 605 slices of a human temporal bone, each with an image dimension of 3,078 $\times 1,942$ pixels and in-plane resolution of $50 \mu \mathrm{m} /$ pixel. As shown in figure $1 \mathrm{~b}$, we 
Kahrs et al.: Freely-Available, True-Color Volume-Rendering Software and

Cryohistology Data Sets for Virtual Exploration of the Temporal Bone Anatomy
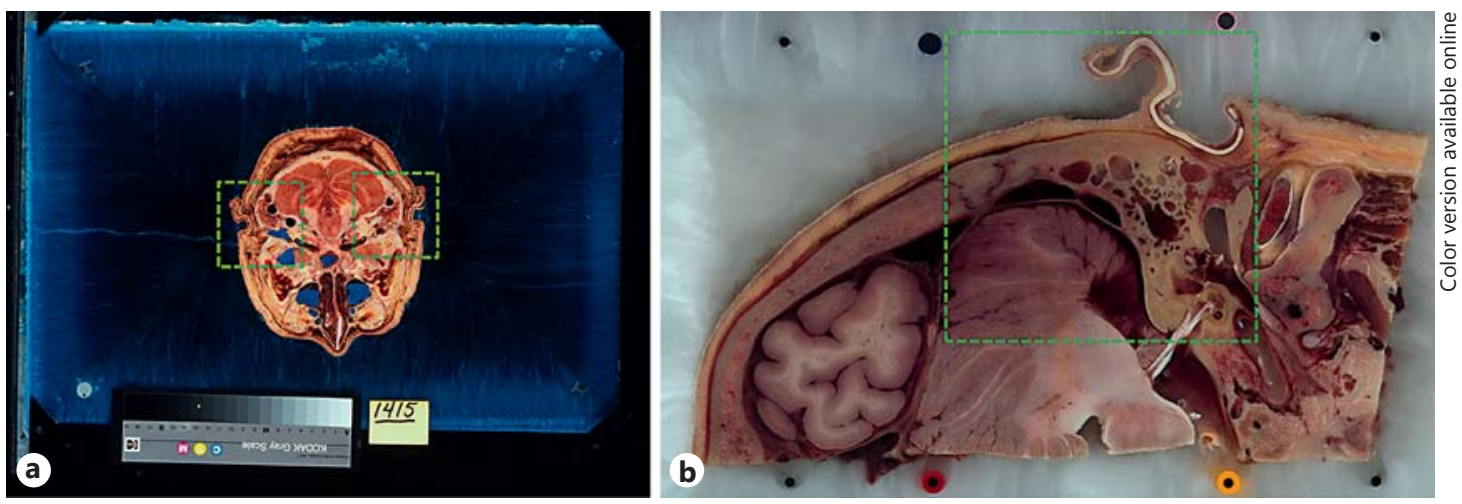

Fig. 1. Exemplary slices of each data set - cropping areas are marked with the dashed lines. a Visible Female Human. b Visible Ear.

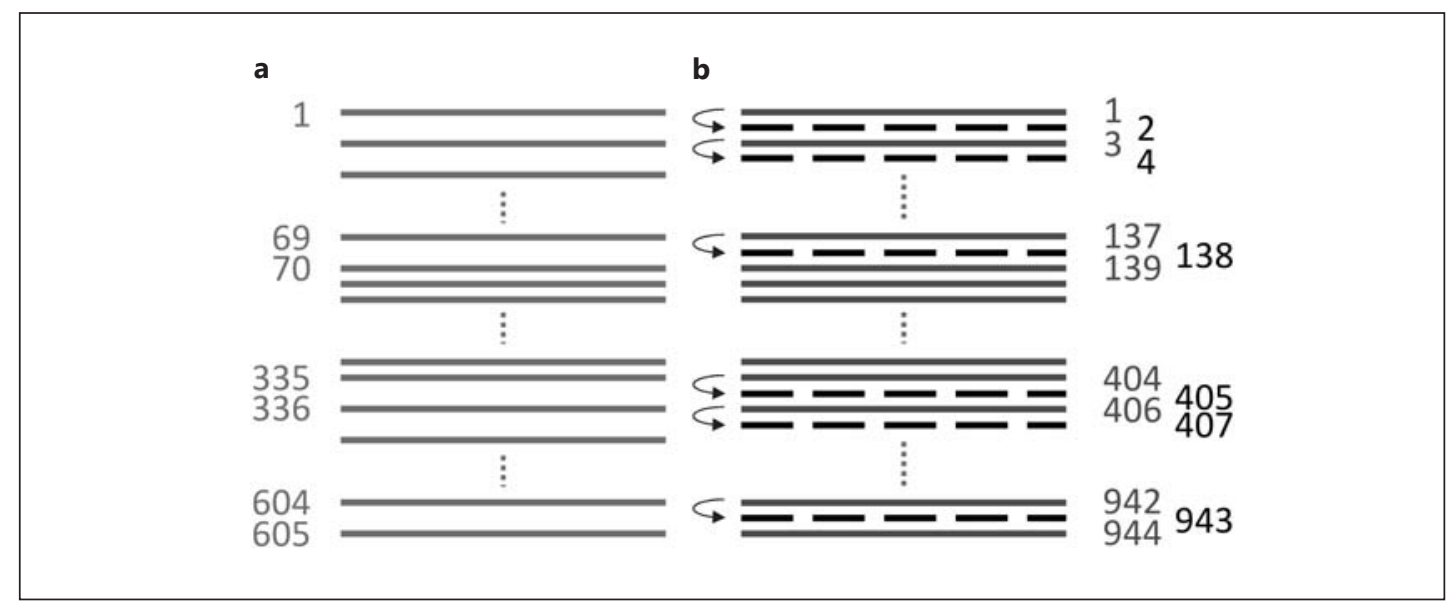

Fig. 2. Image stack of the Visible Ear. The images are symbolized by continuous lines and numbered. The inhomogeneous original data set (a) was enhanced with additional slices (dashed lines) by copying slides and squeezing them in. Between slices No. 70 and 335, the distance of the slices is $50 \mu \mathrm{m}$, between the other slices the original distances were $100 \mu \mathrm{m}$ and are corrected to $50 \mu \mathrm{m}$. The enhanced image stack (b) is renumbered.

cropped the in-plane size of the data set to $1,225 \times 1,225$ pixels and converted the file format to the input format of Vaa3D (i.e. TIFF). The distances between the slices are $50 \mu \mathrm{m}$ for the part encasing the cochlea and $100 \mu \mathrm{m}$ for the other portions of the temporal bone. To have equidistant spacing without compression in the region of the cochlea, duplicate layers were added to regions outside the cochlea so that the whole data set was enlarged to 944 slices with isotropic voxels $\left(50 \times 50 \times 50 \mu^{3}\right)$. To accomplish this, all slices which have $100 \mu \mathrm{m}$ distances are duplicated and inserted immediately after the original slice reducing slice distance to $50 \mu \mathrm{m}$ (fig. 2). This final data set with 1,225 × 1,225 ×944 voxels has a total file size of nearly 4 GB (uncompressed), and the volume covers a region of $6.13 \times 6.13 \times 4.72 \mathrm{~cm}^{3}$. The Visible Ear data set was acquired from a left human temporal bone.

Importantly, while the order of the data sets from the Visible Ear and the Visible Female Human is superior to inferior, the slices need to be sorted descending and renumbered ascending otherwise Vaa3D will not visualize the data set on the correct side of the head (a left ear will be visualized as a right one and vice versa). For all preprocessing of both data sets (cropping, sorting, adding slices, format conversion), we used Irfanview with its Advanced Batch Conversion functions. 
Kahrs et al.: Freely-Available, True-Color Volume-Rendering Software and

Cryohistology Data Sets for Virtual Exploration of the Temporal Bone Anatomy

Table 1. Comparison of the two data sets used for this study

\begin{tabular}{lll}
\hline Original data set & Visible Female Human & Visible Ear \\
\hline Color of embedding material & Blue & Gray \\
Ear & Left and right & Left (flipping for right) \\
Slices inside temporal bone region & $230(1,271-1,500)$ & All $(1-605)$ \\
Original image dimension & $4,096 \times 3,061$ pixels & $3,078 \times 1,942$ pixels \\
Original voxel size & $0.14 \times 0.14 \times 0.33 \mathrm{~mm}^{3}$ & $0.05 \times 0.05 \times 0.05 / 0.1 \mathrm{~mm}^{3}$ \\
Extracted regions for visualization & $512 \times 512 \times 230 \mathrm{voxels}^{3}$ & $1,225 \times 1,225 \times 944 \mathrm{voxels}^{3}$ \\
& $7.04 \times 7.04 \times 7.59 \mathrm{~cm}^{3}$ & $6.13 \times 6.13 \times 4.72 \mathrm{~cm}^{3}$ \\
Crop box coordinates (x, y) & $(2,201,1,029)$ to $(2,712,1,540)$ & $(1,139,98)$ to $(2,363,1,322)$ \\
& $(1,350,1,085)$ to $(1,861,1,596)$ & \\
Automatic sampling factor and resulting & None $(1 \times 1 \times 1)$ & $0.42 \times 0.42 \times 0.27$ \\
$\quad$ voxel size & $0.14 \times 0.14 \times 0.33 \mathrm{~mm}^{3}$ & $0.12 \times 0.12 \times 0.18 \mathrm{~mm}^{3}$ \\
\hline
\end{tabular}

After loading an image stack the triplanar view of the data set appears and a 3D rendering (called 3D view) can be launched. The aforementioned anisotropy factor (e.g. for the Visible Female Human) is needed to adapt the scaling in the $\mathrm{z}$-direction in this view (setting of $\mathrm{z}$-thickness value). The software has multiple options for rendering (e.g. choosing threshold of transparency or opacity) and clipping inside the 3D view. An optimal rendering method for visual exploration is called alpha-value blended, alpha-blending or alpharendering and removes colored regions based on intensity thresholding starting at darker and continuing towards brighter colors.

While the standard rendering resolution of Vaa3D is $512 \times 512 \times 256$ voxels, larger data sets are automatically downsampled. The visualization of more voxels is possible, but to accomplish such requires making a change in Vaa3D's rendering advanced options as well as additional graphics memory on the computer. Table 1 compares the specifications of both data sets.

\section{Results}

When exploring interior structures of both data sets, the Visible Ear appears more natural concerning color and the rendering has more clarity making some structures easy to identify and to follow along their course, e.g. facial nerve, carotid artery, inner auditory canal containing the facial and vestibulocochlear nerves, semicircular canals, ossicles and cochlea. Figure 3 shows the two different volumes used in this study with the triplanar and 3D view using the program's alpha-blending. As described above this alpha-rendering method enables one to show the outer skin surface for the Visible Human (as the skull is embedded in a dark material) and removes the subarachnoid space for the Visible Ear (as this is one of the darkest structures in the data set).

Additionally, Vaa3D allows placement of artificial landmarks represented as spherical markers. They can be placed by the user in one of the views (triplanar or 3D render window) and will be available in all views. This is helpful for localization of structures which are more difficult to follow between slices, e.g. it helped us identify the course of the chorda tympani after initially locating it in one slice following which the spherical markers were visible in 3D views. Figure 4 shows two exemplary images which can be achieved with Vaa3D and the Visible Ear data set while clipping the volume inside the 3D rendering view. A fly-through video is provided as online supplementary material (for all online suppl. material, see www. karger.com/doi/10.1159/000347083). 
Kahrs et al.: Freely-Available, True-Color Volume-Rendering Software and Cryohistology Data Sets for Virtual Exploration of the Temporal Bone Anatomy

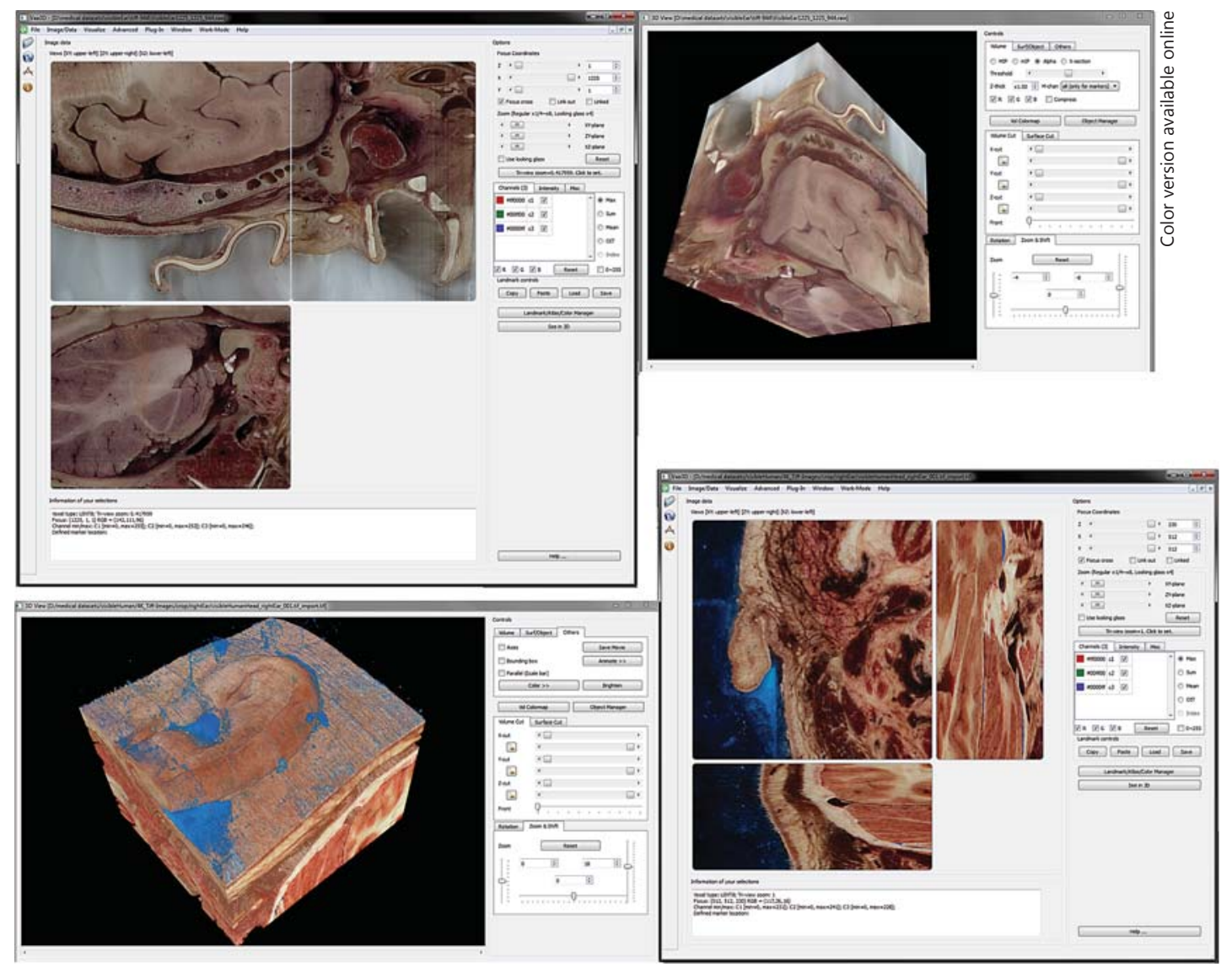

Fig. 3. Both data sets loaded inside Vaa3D and shown in the triplanar and 3D view.
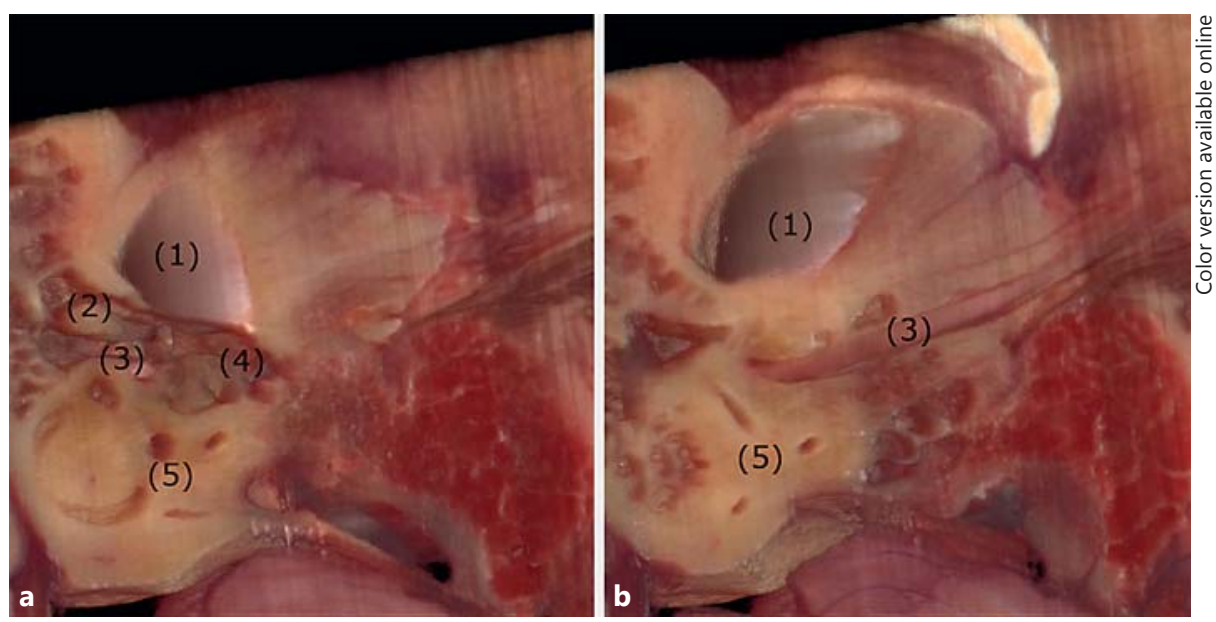

Fig. 4. Two different views inside the Visual Ear data set: more medial (a) and more lateral (b) clipping. Several structures are identified and labeled: (1) the ear canal, filled with the embedding material, (2) ossicles, (3) facial nerve, (4) round window and promontory and (5) semicircular canals. 


\section{Discussion}

Computer-aided visualization of histology image stacks from the temporal bone has been studied for more than 25 years [32]. At present, anatomists and clinicians are encouraged to mentally visualize complex 3D structure using 2-dimensional images. However, modern volume and true-color rendering techniques allow usage of such image stacks for 3D visualization and virtual exploration. To date, their use has been limited due to the need for expensive software as well as the technical knowledge necessary to both set up and run such software. In this context we present instructions for visualization of the complex anatomy of the temporal bone using free software and data sets.

In comparison to the free Visible Ear Simulator software [17,21] or Eaton-Peabody Laboratories 3D Viewer [8], the methods we describe are not limited to specific data sets. Our intent is to allow establishment of a platform by which users may interact with multiple data sets both now and in the future. While at present the Visible Ear data set offers the most complete histological rendering of the temporal bone, future data sets with higher resolution including microscopic images [33, 34] will likely open up further opportunities for virtual ear exploration [35]. Ideally, such data sets should be viewable with a single program such that the user does not need to learn a new program each time a data set becomes available.

While other software packages can carry out similar tasks, Vaa3D is universally available and offers many options suitable for temporal bone exploration. Clinical CT scans can be imported into the software as TIFF image stacks as well. The plug-in interface of Vaa3D offers potential for all kinds of further developments. Haptic feedback as used in other systems [7, $10,21,36$ is not provided in Vaa3D, but may be doable as a future plug-in. Further enhancement of the software which would be beneficial might include the following: automatic removal algorithm of pixels representing embedding material [37], automatic reordering of slices for getting equidistant slices, an automatic method for creating isotropic voxels, interactive removal of tissue by virtual ablation or drilling exceeding the current clipping functions, and creating (e.g. tubular) structures from landmarks and visualizing them as surface renderings directly in the software.

In conclusion, herein we demonstrate a practical solution to understand spatial relationships of the temporal bone using freely available software and data sets. For temporal bone anatomy, the Visible Ear data set is more complete than the Visible Human. This is likely due to the increased sampling in the axial direction, better color calibration, fresher specimen, and more precise image stack alignment. This technique affords an opportunity for virtual exploration. Ongoing work, coupled at registering clinical CT scans with this data set, may allow virtual surgery on individual patients prior to actual intervention.

\section{Acknowledgements}

This work was supported by Award No. R01DC008408 and R01DC010184 (both to R.F.L.) from the National Institute on Deafness and Other Communication Disorders (NIDCD, NIH). L.A.K. thanks support by the German Research Foundation (DFG) within the project KA 2975/2-1. L.A.K. also thanks Jessica Burgner for help during exploration of the Visible Female Human. The content is solely the responsibility of the authors and does not necessarily represent the official views of NIDCD, NIH or DFG.

\section{Disclosure Statement}

R.F.L. is consultant for Cochlear and is on the Surgical Advisory Boards of MED-EL and Ototronix. 


\begin{tabular}{l|l}
\hline ORL $2013 ; 75: 46-53$ \\
\hline DOI: $10.1159 / 000347083$ \\
\hline
\end{tabular}

Kahrs et al.: Freely-Available, True-Color Volume-Rendering Software and

Cryohistology Data Sets for Virtual Exploration of the Temporal Bone Anatomy

\section{References}

1 Hoeffner EG, Mukherji SK, Gandhi D, Gomez-Hassan D, Gujar S, Ibrahim M, Parmar H: Temporal Bone Imaging, ed 1. New York, Thieme, 2008, p 244.

2 Noble JH, Dawant BM, Warren FM, Labadie RF: Automatic identification and 3D rendering of temporal bone anatomy. Otol Neurotol 2009;30:436-442.

3 Fennessy BG, O'Sullivan P: Establishing a temporal bone laboratory: considerations for ENT specialist training. Ir J Med Sci 2009;178:393-395.

- 4 George AP, De R: Review of temporal bone dissection teaching: how it was, is and will be. J Laryngol Otol 2010; 124:119-125.

- 5 Walter T, Shattuck DW, Baldock R, Bastin ME, Carpenter AE, Duce S, Ellenberg J, Fraser A, Hamilton N, Pieper S, Ragan MA, Schneider JE, Tomancak P, Hériché JK: Visualization of image data from cells to organisms. Nat Methods 2010;7(suppl):S26-S41.

- 6 Clifton N, Klingmann C, Khalil H: Teaching otolaryngology skills through simulation. Eur Arch Otorhinolaryngol 2011;268:949-953.

- 7 Wiet GJ, Stredney D, Kerwin T, Hittle B, Fernandez SA, Abdel-Rasoul M, Welling DB: Virtual temporal bone dissection system: Development and Testing. Laryngoscope 2012;122(suppl 1):S1-S12.

- 8 Wang H, Merchant SN, Sorensen MS: A downloadable three-dimensional virtual model of the visible ear. ORL 2007;69:63-67.

9 Kockro RA, Hwang PYK: Virtual temporal bone: an interactive 3-dimensional learning aid for cranial base surgery. Neurosurgery 2009;64(suppl 2):216-229.

$>10$ Sørensen MS, Mosegaard J, Trier P: The visible ear simulator: a public PC application for GPU-accelerated haptic 3D simulation of ear surgery based on the visible ear data. Otol Neurotol 2009;30:484-487.

$\checkmark 11$ Tolsdorff B, Petersik A, Pflesser B, Pommert A, Tiede U, Leuwer R, Höhne KH: Individual models for virtual bone drilling in mastoid surgery. Comput Aided Surg 2009;14:21-27.

12 Lee DH, Chan S, Salisbury C, Kim N, Salisbury K, Puria S, Blevins NH: Reconstruction and exploration of virtual middle-ear models derived from micro-CT datasets. Hear Res 2010;263:198-203.

13 Wiet GJ, Stredney D, Wan D: Training and simulation in otolaryngology. Otolaryngol Clin North Am 2011;44: 1333-1350, viii-ix.

14 Tiede U, Schiemann T, Hohne KH: High quality rendering of attributed volume data. Proceedings of Visualization '98, 1998, Research Triangle Park, pp 255-262..

$>15$ Heng PA, Zhang SX, Xie YM, Wong TT, Chui YP, Cheng CY: Photorealistic virtual anatomy based on Chinese Visible Human data. Clin Anat 2006;19:232-239.

-16 Robb RA, Hanson DP: Biomedical image visualization research using the Visible Human datasets. Clin Anat 2006;19:240-253.

17 Trier P, Noe KØ, Sørensen MS, Mosegaard J: The visible ear surgery simulator. Stud Health Technol Inform 2008; 132:523-525.

18 Roy D, Steyer GJ, Gargesha M, Stone ME, Wilson DL: 3D cryo-imaging: a very high-resolution view of the whole mouse. Anat Rec (Hoboken) 2009;292:342-351.

19 Handschuh S, Schwaha T, Metscher BD: Showing their true colors: a practical approach to volume rendering from serial sections. BMC Dev Biol 2010;10:41.

20 Dai J-X, Chung MS, Qu R-M, Yuan L, Liu S-W, Shin DS: The Visible Human Projects in Korea and China with improved images and diverse applications. Surg Radiol Anat 2012;34:527-534.

21 Kostack K: The Visible Human in real-time with blender. 2009. http://vimeo.com/4310269 and http://www. youtube.com/watch?v=6Yn-D5LnQjI (accessed September 1, 2012].

-22 Long F, Zhou J, Peng H: Visualization and analysis of 3D microscopic images. PLoS Comput Biol 2012; 8:e1002519.

-23 Ackerman MJ, Spitzer VM, Scherzinger AL, Whitlock DG: The Visible Human data set: an image resource for anatomical visualization. Medinfo 1995;8:1195-1198.

$>24$ Zhang SX, Heng PA, Liu ZJ, Tan LW, Qiu MG, Li QY, Liao RX, Li K, Cui GY, Guo YL, Yang XP, Liu GJ, Shan JL, Liu JJ, Zhang WG, Chen XH, Chen JH, Wang J, Chen W, Lu M, You J, Pang XL, Xiao H, Xie YM: Creation of the Chinese Visible Human data set. Anat Rec B New Anat 2003;275:190-195.

25 Park JS, Chung MS, Hwang SB, Shin B-S, Park HS: Visible Korean Human: its techniques and applications. Clin Anat 2006;19:216-224.

-26 Sørensen MS, Dobrzeniecki AB, Larsen P, Frisch T, Sporring J, Darvann TA: The Visible Ear: a digital image library of the temporal bone. ORL 2002;64:378-381.

27 Qiu M-G, Zhang S-X, Liu Z-J, Tan L-W, Li Q-Y, Li K, Wang Y-S, Deng JH, Tang ZS: Visualization of the temporal bone of the Chinese Visible Human. Surg Radiol Anat 2004;26:149-152.

28 Jang HG, Chung MS, Shin DS, Park SK, Cheon KS, Park HS, Park JS: Segmentation and surface reconstruction of the detailed ear structures, identified in sectioned images. Anat Rec (Hoboken) 2011;294:559-564.

29 Peng H, Ruan Z, Atasoy D, Sternson S: Automatic reconstruction of 3D neuron structures using a graphaugmented deformable model. Bioinformatics 2010;26:i38-i46.

- 30 Peng H, Ruan Z, Long F, Simpson JH, Myers EW: V3D enables real-time 3D visualization and quantitative analysis of large-scale biological image data sets. Nat Biotechnol 2010;28:348-353. 
31 Ackerman MJ, Yoo TS: The Visible Human data sets (VHD) and Insight Toolkit (ITk): experiments in open source software. Proc Annu Symp Am Med Inform Assoc, Washington, 2003, p 773.

-32 Harada T, Ishii S, Tayama N: Three-dimensional reconstruction of the temporal bone from histologic sections. Arch Otolaryngol Head Neck Surg 1988;114:1139-1142.

33 Eckardt F, Rau T, Lenarz T, Majdani O: Verfahren der punktbasierten Fusionierung hochaufgelöster Detailbilder mit histologischen Schichtdatensätzen zur Darstellung der Weichgewebsanatomie des humanen Innenohres. Proc Annu Meet German Soc Comput Robot-Aided Surg, Magdeburg, 2011.

34 Rau TS, Hussong A, Herzog A, Majdani O, Lenarz T, Leinung M: Accuracy of computer-aided geometric 3D reconstruction based on histological serial microgrinding preparation. Comput Methods Biomech Biomed Engin 2011;14:581-594.

-35 Neri E, Caramella D, Battolla L, Cosottini M, Scasso CA, Bruschini P, Pingitore R, Bartolozzi C: Virtual endoscopy of the middle and inner ear with spiral computed tomography. Am J Otol 2000;21:799-803.

36 Morris D, Sewell C, Blevins N, Barbagli F, Salisbury K: A collaborative virtual environment for the simulation of temporal bone surgery. Proc 7th Int Conf Med Image Comput Computer-Assisted Interv, Saint-Malo, 2004, Berlin, Springer, 2004, vol 3217, pp 319-327.

37 Matuszek S: Volume rendering of the photographic Visible Human data set. 1998. http://matuszek.net/development/graphics/visible/ (accessed September 1, 2012). 